\title{
Parametric Study of Magnus Wind Turbine with Spiral Fins using BEM Approach
}

\author{
R. Mdouki ${ }^{\dagger}$ \\ Environmental Laboratory, Larbi Tebessi University, Constantine Avenue, Tebessa, 12002, Algeria \\ †Corresponding Author Email: ramzi.mdouki@univ-tebessa.dz
}

(Received May 8, 2020; accepted November 15, 2020)

\begin{abstract}
The aim of this work is to carry out an aerodynamic analysis to assess the power and identify the characteristics of the horizontal axis Magnus type wind turbine with spiral fins. A parametric study is achieved to analyze the effects of the different influence parameters such as the turbine tip speed ratio, the cylinder spinning ratio, the blade aspect ratio and the cylinder hub-tip ratio. The analysis approach adopted in this study is the Blade Element Momentum BEM using the experimental lift coefficient data for the configuration of spinning cylinders with spiral fins. In this analysis, losses are not taken in consideration. Both axial and angular interference coefficients are evaluated for this type of wind turbine. The former is assessed by solving a quadratic equation and the latter is calculated from a classic formulation including the term of spinning. An iterative process is followed to achieve this task. Concerning the obtained results, the aerodynamic characteristics of the Magnus wind turbine, analyzed in this study, provide some elucidation to lead a successful preliminary design of this novel type of machine.
\end{abstract}

Keywords: Horizontal axis wind turbine; Magnus effect; Spiral fins; Blade element momentum; Parametric study.

\section{NOMENCLATURE}

A cross-sectional area

AR cylinder aspect ratio

a axial induction coefficient

$a^{\prime} \quad$ angular induction coefficient

$B \quad$ extensive parameter

$b \quad$ intensive parameter

$C_{D} \quad$ drag coefficient

$C_{L} \quad$ lift coefficient

$C_{P} \quad$ power coefficient

$C_{N}$ normal coefficient

$C_{T} \quad$ thrust coefficient

$D$ rotor diameter

$d \quad$ cylinder diameter

$\mathrm{Nb} \quad$ number of blades

$P \quad$ power

$p \quad$ pressure

Re Reynolds number

$\begin{array}{ll}R h & \text { hub radius } \\ R h t & \text { hub tip ratio } \\ R t & \text { tip radius } \\ r_{c} & \text { cylinder radius } \\ V & \text { absolute velocity } \\ V_{\infty} & \text { freestream velocity } \\ V_{w} & \text { wake velocity } \\ W & \text { relative velocity } \\ & \\ \phi & \text { relative flow angle } \\ \Omega & \text { rotor angular velocity } \\ \lambda & \text { tip speed ratio } \\ \lambda_{\mathrm{r}} & \text { local speed ratio } \\ \omega & \text { cylinder spin } \\ \varpi & \text { spinning cylinder ratio } \\ \sigma_{\mathrm{r}} & \text { local solidity }\end{array}$

particularly the wind energy. This interest is stem from wind source features; clean, sustainable and renewable source which represents an alternative to fossil fuels and a remedy for greenhouse gas
It is worth noting that the renewable energy has seen an enormous interest in this modern era, 
effects. In fact, the wind energy is harnessed via either horizontal axis wind turbine HAWT or vertical axis wind turbine VAWT. Regardless the type of wind turbine, the blade represents the key element used to transform the wind kinetic energy into shaft work. This energy conversion is carried out via the aerodynamic forces acting on the blades by the wind flow, thus two main aims behind this procedure are appeared; the first is to maximize the wind turbine output power and obtain a satisfactory levels of performance, and the second represents the ability to absorb wind energy and push the turbine to work under low values of wind speed.

One of the shortcomings that suffers from the Algerian territory is the low wind speed. In fact, the maximum threshold to use the wind turbines reaches $7 \mathrm{~m} / \mathrm{s}$ in the region of Adrar, (Himri et al. 2010). In front of this situation, the use of conventional wind turbine reveals inefficience. Fortunately, the newer Magnus wind turbine which is appeared recently in the wind energy field proves its benefits especially the better efficiency for low wind speed compared to the conventional bladestype wind turbine (Murakami 2007). Therefore, it is important to provide some insights to elucidate the power performance and the characteristics of the horizontal axis Magnus wind turbine in order to find its position in the Algerian territory and promote its application as a generator of electricity or device to pump water. According to Seifert in his review, the one barrier for using Magnus rotors is the lack of design information of the rotor aeroplane lifting force (Seifert 2012). The majority works either analytical (Luo et al. 2011; Sedaghat 2014) or numerical (Xiaojing et al. 2012; Aneesh et al. 2016), and experimental (Bychkov et al. 2007, 2008a, b; Marzuki et al. 2015) which have been conducted in this area use a smooth spinning cylinders and show that the small wind turbine applications are promising, contrary to the large ones which need more experimental data to guarantee their use. In this work, a parametric study is carried out to analyze the effects of the different influence parameters such as the turbine tip speed ratio, the cylinder spinning ratio, the blade aspect ratio and the cylinder hub-tip ratio. Using the analytical-numerical blade element momentum approach with only experimental values of lift coefficient applied with the Reynolds number of 1.9 $10^{4}$ and spinning ratio located in the range $0-1.1$, respectively. The expressions of axial and angular induction factors are obtained for the Magnus type wind turbine without tip and hub losses. The aerodynamic characteristics of the Magnus wind turbine are analyzed in order to provide some elucidations and therefore arrive to perform a well preliminary design of this new type of horizontal axis wind turbine, (Fig.1).

\section{EXPERIMENTAl DATA OF Lift For ROTARY CYLINDER WITH SPIRAL FINS}

Both aerodynamic coefficients lift and drag represent the key data required in the blade element momentum method. These aerodynamic coefficients depend essentially, for the flow around rotary columns, on the spinning ratio $\varpi=\omega d / 2 V_{\infty}$ and the Reynolds number $R e=V_{\infty} d / v$. Basing on the experimental study conducted by Nobuhiro et al. (Masaya et al. 2018; Murakami et al. 2016) the measurements were conducted to obtain the lift for column diameter $d=0.07 \mathrm{~m}$, mainstream velocity $V_{\infty}=4 \mathrm{~m} / \mathrm{s}, \quad$ Reynolds number $R e=1.910^{4}$ and spinning ratio range $0 \leq \varpi \leq 1.1$. The Fig. 2 shows only the evolution of the lift coefficient depending on the spinning ratio for three models (no fin, straight fin and spiral fin) compared to the potential model, while the drag coefficient is not taken into consideration. It is clear from the figure that the configurations with fins is more performant than without fins. In particular, the rotary cylinder with spiral fins marks its high potential which mimics the ideal flow behaviour $C_{L}=2 \pi \varpi$ when $\varpi$ is less than 1. Therefore, in our study the spinning cylinders with spiral fins are testified as blades in the horizontal wind turbine which the corresponding experimental curve of the lift is fitted and a correlation with polynomial fit is deduced as following:

$$
\begin{aligned}
& C_{L}=2.644+1.312 \varpi-0.6055 \varpi^{2} \\
& -0.325 \varpi^{3}+0.02584 \varpi^{4}+0.1544 \varpi^{5}
\end{aligned}
$$

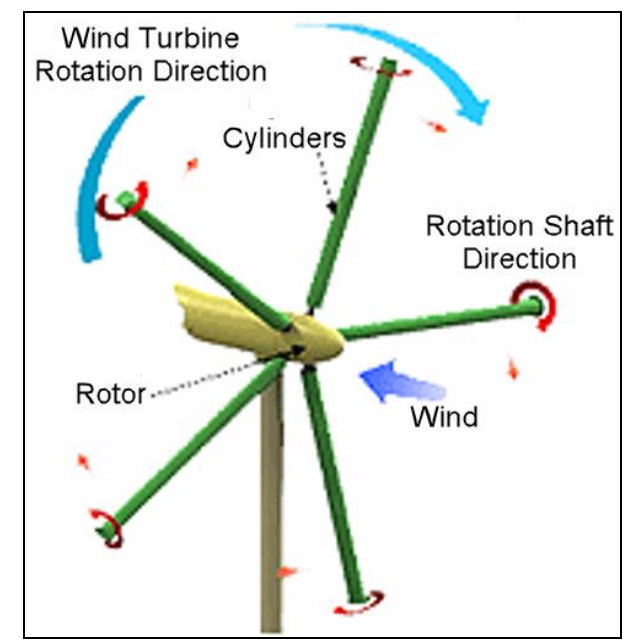

Fig. 1. Magnus type wind turbine with spiral fins.

\section{DISC ACTUATOR MODEL}

Using the concept of actuator disk, the rotor of the Magnus type horizontal axis wind turbine is considered as a disk with infinite permeability, infinite number of rotating circular cylinders and negligible thickness. The only task of the disk is to extract the energy from the air which flows with inviscid, incompressible and steady behavior in a divergent stream tube composed of two parts with lateral surface $(S)$, (fig.3). Both upwind and downwind regions are separated by the disk $(d)$ and therefore limited in the axial direction by the stations $\left(\infty, d^{-}\right)$and $(d+, w)$, respectively. Applying 
the Reynolds transport theory over the control volume $(C V)$ illustrated in the undermentioned figure, (Branlard 2017; Sørensen 2016; Sedaghat 2014).

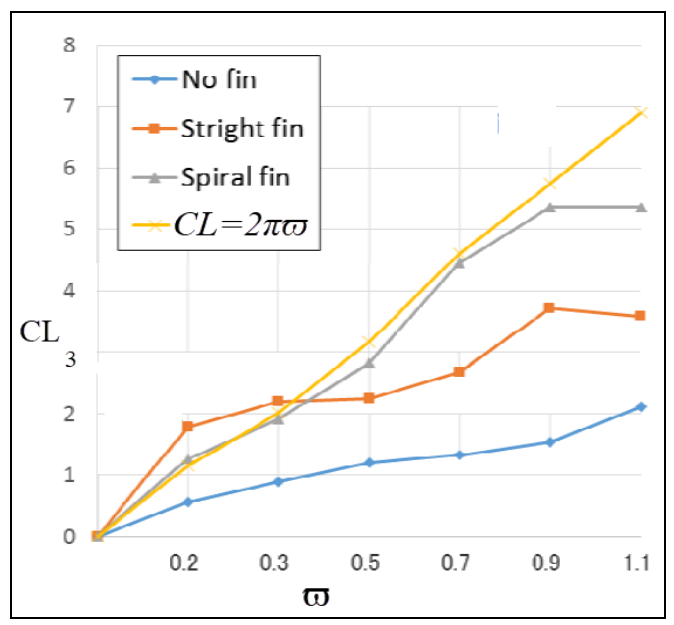

(a)

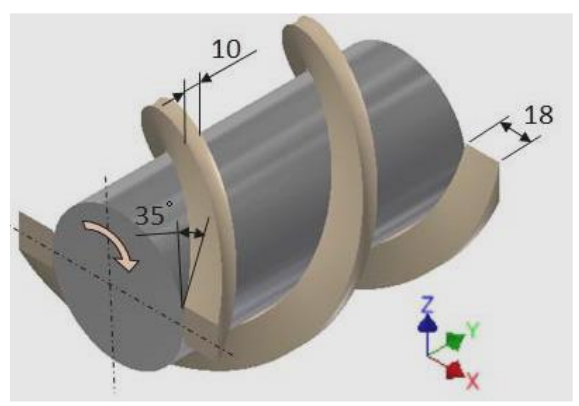

(b)

Fig. 2. Rotating cylinders with spiral fins: (a) lift coefficient curve, (b) spiral fins type.

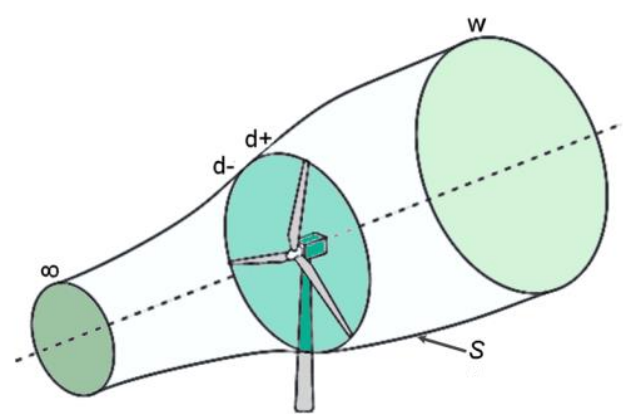

Fig. 3. Actuator disk model.

In steady regime, an important integral equation which deals with the different conservation laws; mass, linear momentum, angular momentum and energy, is expressed as:

$\frac{d B_{s y s}}{d t}=\int_{C S} b \rho \overrightarrow{V d} \vec{A}$

Where: $B_{s y s}$ and $b$ are two parameters which take different forms depending the conservation equation.

\subsection{Conservation of Mass}

This principle leads to the continuity equation. The integral formulation of the mass conservation is written as:

$\int_{C S} \rho \overrightarrow{V d} \vec{A}=0$

In other word, the mass flow rate is the same at all cross sections. Considering the axial direction, the actuator disk produces an induced axial velocity $-a V_{\infty}$ added to the upstream velocity $V_{\infty}$, hence the velocity at the disk take the form:

$V_{d}=V_{\infty}(1-a)$

The velocity coefficient $C_{v}$ is expressed as:

$C_{V}=\frac{V_{d}}{V_{\infty}}=1-a$

\subsection{Conservation of linear momentum}

The Conservation of linear momentum equation is written in its integral formulation as:

$\int_{C S} \vec{V} \rho \overrightarrow{V d} \vec{A}=\sum \vec{F}$

In fact, the reaction of the thrust force $R=-T$ is the concerned force applied in the Eq. (6) between the inlet $(\infty)$ and the outlet $(w)$ with the minus sign after projection of the reaction force in the axial direction, thus:

$T=q_{m}\left(V_{\infty}-V_{w}\right)$

Using the Bernoulli equation in two regions; between the far upstream $(\infty)$ and just before the disk $(d-)$, and from just after the disk $(d+)$ to the far downstream $(w)$; the thrust force is written as:

$T=\frac{1}{2} \rho A_{d}\left(V_{\infty}^{2}-V_{w}^{2}\right)$

Thus, the thrust coefficient $C_{T}$ is defined in term of the factor $a$ as:

$C_{T}=\frac{2 T}{\rho V_{\infty}^{2} A_{d}}=4 a(1-a)$

\subsection{Conservation of Energy}

The principle of energy conservation may be used by applying the first law of thermodynamic to a system which leads to write, under the assumptions cited previously in the model of the actuator disk, the following equation:

$\dot{W}_{\text {shaft }}-\int_{C S} p \overrightarrow{V d} \vec{A}=\int_{C S}\left(V^{2} / 2\right) \rho \vec{V} d \vec{A}$

In wind turbine, the power $P=-W_{\text {shaft }}$ provided by the shaft is :

$P=\frac{1}{2} q_{m}\left(V_{\infty}^{2}-V_{w}^{2}\right)$

The power coefficient in term of the axial induction factor $a$ is written as: 
$C p=4 a(1-a)^{2}$

\subsection{Conservation of Angular Momentum}

Applying the moment of the Newton's second law, the integral equation of the angular moment conservation takes the form:

$\int_{C S}(\vec{r} \wedge \vec{V}) \rho \overrightarrow{V d} \vec{A}=\sum M$

While the wake zone is qualified with rotation, the axial interference factor $a$ is used jointly with the angular interference factor $a^{\prime}$ in the process of design and performance analysis of the wind turbine. The angular induction factor $a$ ' is appeared in the Magnus type HAWT under the effect of blades rotation and cylinders spinning. This factor will be addressed later. In the simple case, without the spinning of cylinders, the rotor torque $Q$ and the associated dimensionless coefficient $C_{Q}$ are expressed, respectively, as:

$$
Q=4 \pi \rho V_{\infty} \Omega \int_{R_{h}}^{R_{t}} a^{\prime}(1-a) r^{3} d r
$$

and

$$
C_{Q}=\frac{2 Q}{\rho V_{\infty}^{2} A_{d} R}=\frac{8}{\lambda^{3}} \int_{R_{h}}^{R_{t}} a^{\prime}(1-a) \lambda_{r} d \lambda_{r}
$$

\section{Blade Element Momentum APPROACH}

Both theories linear and angular momentum lead to define the performance parameters of wind turbine as a function of the induction factors $a$ and $a^{\prime}$. In fact, the momentum theory is considered as a global method which considers the rotor as an actuator disk where the aerodynamic aspect of the blades is not taken into account. In contrast, the blade element theory introduces the notion of the design of particular blade and the aerodynamic forces produced on it. In the two following sections, the first represents a detailed description of the blade element theory that corresponds to the spinning cylinders instead of the conventional blades while the second illustrates the combination of both approaches momentum and blade element.

\subsection{Blade Element Theory}

The concept of this theory is based on the division of the rotor into a set of annular strips that intersect with the cylindrical columns to give the blade elements. In Magnus type HAWT the blades are represented by elemental cylinders located at a radial station $r$ with a thickness dr (Fig. 4). The lift and drag forces for rotating cylinders with the velocity triangles are presented in the Fig. 5. The relative velocity $W$ has two components, the axial velocity $(1-a) V_{\infty}$ and the circumferential velocity $\operatorname{sr}\left(1+a^{\prime}\right)-\omega r_{c} / 2$.

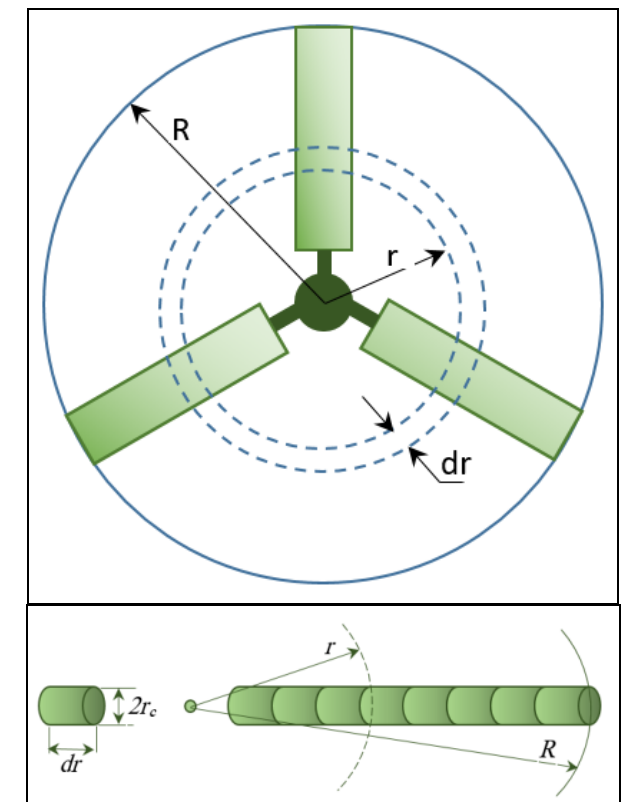

Fig. 4. Cylindrical column and blade element

Let consider a Magnus rotor with $N b$ rotating circular cylinders of tip and hub radii $R t$ and $R h$, respectively, operates under angular velocity $\Omega$, cylinder spin $\omega$ and upstream wind speed $V_{\infty}$, (Sedaghat 2014). The tangential velocity $\Omega r$ of the blade element is increased, on the one hand, by the angular induced velocity $a$ ' $\Omega r$ due to the wake and is decreased, in other hand, by the angular induced velocity $\omega r_{d} / 2$ due to the cylinder spinning. Therefore, the net tangential flow velocity undergo by the cylinder element is $\Omega r\left(1+a^{\prime}\right)-\omega r_{d} / 2$. Thus, the resultant relative velocity at the elemental cylinder is, (Fig.5):

$W=\sqrt{V_{\infty}^{2}(1-a)^{2}+\left[\Omega r\left(1+a^{\prime}\right)-\omega r_{c} / 2\right]^{2}}$

Which is oriented under the relative flow angle $\phi$ with respect to rotation plane such as:

$\phi=\tan ^{-1}\left(\frac{1-a}{\left(1+a^{\prime}\right) \lambda_{r}-\varpi / 2}\right)$

Where, $\lambda_{r}=\Omega r / V_{\infty}$ is the local speed ratio. The lift of each annular portion, normal to $W$, is

$d L=\frac{N b}{2} \rho W^{2}\left(2 r_{c}\right) C_{L} d r$

And the drag force parallel to $W$ is

$d D=\frac{N b}{2} \rho W^{2}\left(2 r_{c}\right) C_{D} d r$

Both normal and tangential forces $d F_{N}$ and $d F_{T}$ that contributes to produce the thrust and the torque on the rotor, respectively, are

$$
\begin{aligned}
d F_{N} & =d L \cos \phi+d D \sin \phi \\
& =N b \rho W^{2} r_{c}\left(C_{L} \cos \phi+C_{D} \sin \phi\right) d r
\end{aligned}
$$




$$
\begin{aligned}
d F_{T} & =d L \sin \phi-d D \cos \phi \\
& =N b \rho W^{2} r_{c}\left(C_{L} \sin \phi-C_{D} \cos \phi\right) d r
\end{aligned}
$$

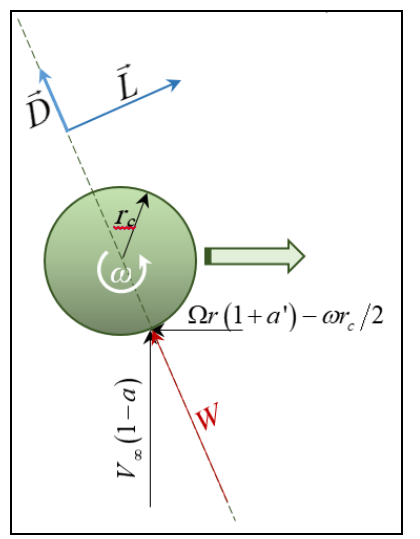

Fig. 5. Velocity triangle for spinning columns.

The forces $d F_{N}$ and $d F_{T}$ can be expressed in term of both coefficients $C_{N}$ and $C_{T}$, respectively:

$$
d F_{N}=N b \rho W^{2} r_{c} C_{N} d r
$$

and

$$
d F_{T}=N b \rho W{ }^{2} r_{c} C_{T} d r
$$

Thus, the elemental torque of each ring is

$$
\begin{aligned}
d M & =r d F_{T} \\
& =N b \rho W^{2} r_{c}\left(C_{L} \sin \phi-C_{D} \cos \phi\right) r d r
\end{aligned}
$$

And the elemental extracted power is

$$
\begin{aligned}
d P & =\Omega d M \\
& =N b \rho W^{2} r_{c}\left(C_{L} \sin \phi-C_{D} \cos \phi\right) \Omega r d r
\end{aligned}
$$

\subsection{Combination of Momentum and Blade Element Theories}

Combining both previous developed theories; momentum and blade element, the rotor should be divided into annular strips and the elemental lift and drag forces acting on blade elements are set equal to the change of the momentum of the air which passes through the element swept area. Ignoring the radial interaction between the air and the rotor, the blade element momentum BEM leads to write for the linear momentum

$$
\begin{aligned}
d F_{N} & =N b \rho W{ }^{2} r_{c}\left(C_{L} \cos \phi+C_{D} \sin \phi\right) d r \\
& =4 \pi \rho\left[V_{\infty}^{2} a(1-a)+\frac{1}{4}\left(2 a^{\prime} \Omega r-\omega r_{c}\right)^{2}\right]
\end{aligned}
$$

After rearrangements

$$
\begin{aligned}
& \frac{W^{2}}{V_{\infty}^{2}} N b\left(\frac{2 r_{c}}{R}\right)\left(C_{L} \cos \phi+C_{D} \sin \phi\right) d r= \\
& \frac{8 \pi r}{R}\left[a(1-a)+\frac{1}{4 V_{\infty}^{2}}\left(2 a^{\prime} \Omega r-\omega r_{c}\right)^{2}\right]
\end{aligned}
$$

For the angular momentum, the elemental torque is determined as follows

$$
\begin{aligned}
d M & =r d F_{T} \\
& =N b \rho W^{2} r_{c}\left(C_{L} \sin \phi-C_{D} \cos \phi\right) r d r \\
& =2 \pi \rho V_{\infty}(1-a)\left(2 a^{\prime} \Omega r-\omega r_{c}\right) r^{2} d r
\end{aligned}
$$

After simplifications

$$
\begin{aligned}
& \frac{W^{2}}{V_{\infty}^{2}} N b\left(\frac{2 r_{c}}{R}\right)\left(C_{L} \sin \phi-C_{D} \cos \phi\right) d r= \\
& \frac{4 \pi r}{R V_{\infty}}(1-a)\left(2 a^{\prime} \Omega r-\omega r_{c}\right)
\end{aligned}
$$

Defining the local solidity, $\sigma_{r}=c N_{b} / 2 \pi r=r_{c} N_{b} / \pi r$, the Eq. (27) leads to find the axial induction factor by solving the following second degree equation

$$
C_{1} a^{2}+C_{2} a+C_{3}=0
$$

Where:

$$
\begin{aligned}
& C_{1}=1+\frac{\sigma_{r} C_{N}}{4 \sin ^{2} \phi}-\operatorname{cotg}^{2} \phi \\
& C_{2}=-\frac{\sigma_{r} C_{N}}{2 \sin ^{2} \phi}+2 \operatorname{cotg}^{2} \phi-2 \lambda_{r} \operatorname{cotg} \phi-1 \\
& C_{3}=\frac{\sigma_{r} C_{N}}{4 \sin ^{2} \phi}-\operatorname{cotg}^{2} \phi+2 \lambda_{r} \operatorname{cotg} \phi-\lambda_{r}^{2}
\end{aligned}
$$

The axial interference factor takes the positive value among the two roots

$$
a_{1,2}=\frac{-C_{2} \pm \sqrt{\delta}}{2 C_{1}}
$$

Where:

$\delta=C_{2}^{2}-4 C_{1} C_{3}$

Concerning the angular interference factor, its value is found from the equation

$$
a^{\prime}=\frac{1}{\frac{4 \sin \phi \cos \phi}{\sigma_{r} C_{T}}-1}+\frac{\varpi}{2 \lambda_{r}}
$$

In fact, the values of the induction factor $a$ and $a$, can be calculated if only the relative angle $\phi$ is known. Thus the Eq. (17) is needed to solve the system.

\subsection{Algorithm}

Based on the developed equations from (16) to (36), an iterative process should be performed to obtain a solution for this system including the unknowns $a$, $a^{\prime}$ and $\phi$. Calculating the lift and drag coefficients (in this study the drag is not taken in consideration), the following set of steps should be followed, (Branlard 2017; Sørensen 2016; Tahani and Moradi 2016):

1. Give the input data; $V_{\infty}, R h t, A R, N b$,, and $\lambda$, 
2. Assume initial values $a=1 / 3$ and $a^{\prime}=0$,

3. Calculate the angle $\phi$ using "Eq. (17),"

4. Determine the lift coefficient $C_{L}$ from the fitted experimental curve "Eq. (1),",

5. Calculate the new values of both induction factors; $a$ and $a$ ' using, respectively, "Eqs. (34) and (36),"

6. Repeat the previous steps until the values of the induction factors converge.

7. Calculate the aerodynamic forces on the local portion of the cylinder

\subsection{Code Validation}

In order to validate the developed BEM code which is intended to design and analyse the Magnus type wind turbine with spiral fins, the experimental data that can be found in the patents published by the inventors Murakami in Japan and Kheirandish in Iran (Kheirandish 2016; Murakami 2007) are used to confront them with the BEM results for a rotor configuration identified by a diameter equal to $2 \mathrm{~m}$ and 5 spiral finned rotating columns in the speed wind range between 1 and $12 \mathrm{~m} / \mathrm{s}$. The comparison is illustrated in the Fig. 6. The BEM code leads to a good agreement with the experimental data cited in (Murakami 2007) and the results are in reasonable range and values, especially for wind speed less than $8 \mathrm{~m} / \mathrm{s}$.

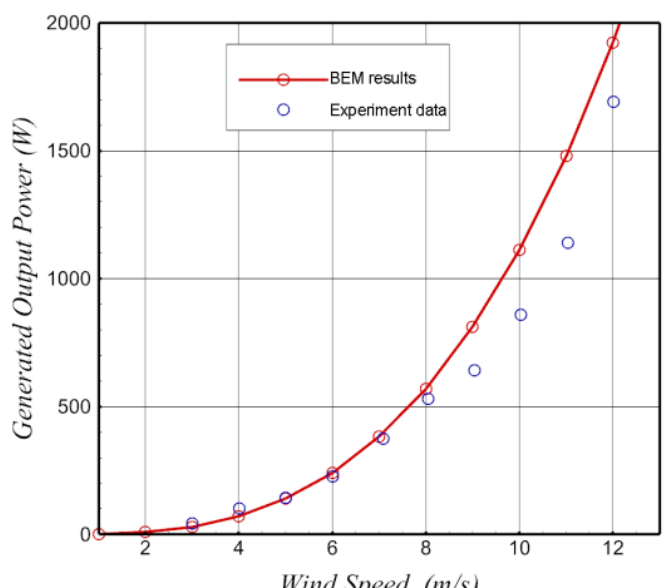

Fig. 6. Comparison between experimental data and BEM results for Magnus type wind turbine with spiral fins and diameter of $2 \mathrm{~m}$.

\section{RESUlts AND Discussion}

In this part of the paper, a parametric study is conducted where the effects of four parameters are analyzed; the blade aspect ratio $A R$, the cylinder spinning ratio $\varpi$, the turbine tip speed ratio $\lambda$, and the hub-tip ratio $R h t$ of blades. Each influence parameter is examined independently and the power coefficient is adopted to assess the performance of the Magnus wind turbine for different number of blades.

The calculations were carried out with the developed BEM code for the Magnus type wind turbine under the following design parameters:

- The cylinder spinning ratio varies from 0.1 to 1.1 ,

- The aspect ratio is between $3 \leq A R \leq 27$,

- The tip speed ratio varies from 3 to 10 ,

- The number of blades $1 \leq N b \leq 10$,

- The hub-tip ratio takes the value $R h t=0.2$,

- The rotor radius is selected as $R t=3 \mathrm{~m}$,

- The freestream wind velocity $V_{\infty}=5 \mathrm{~m} / \mathrm{s}$.

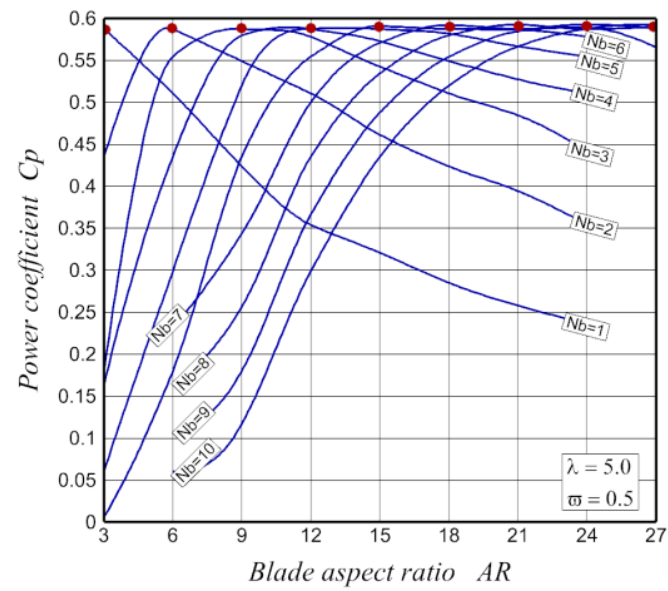

Fig. 7. $C p$ versus $A R$ for ten values of number of blades with $\lambda=5$ and $\varpi=0.5$.

In the first step, the aim is to find the best value of the column aspect ratio by giving both kinematic parameters, tip speed ratio and spinning cylinder ratio, the values $\lambda=5$ and $\varpi=0.5$, respectively. Concerning the geometric parameter, the aspect ratio of the cylinders $A R$ is varied from 3 to 27 . In Fig. 7, the power coefficient curves are laid out with respect to the number of blades $N b$. The latter takes ten values vary from 1 to 10 blades. Each curve marks a maximum threshold which corresponds to the Betz limit and therefore yields the optimal aspect ratio.

The optimum values of aspect ratio for each blades number are summarized in the table 1 . From the results, a linear relationship between the cylinder aspect ratio and the number of blades can be expressed as follows:

For $\mathrm{Nb} \leq 5$ :

$A R=3 N b$

For $N b \geq 5$.

$A R=3 N b-3$ 
Table 1 Summary of optimal $N b$ for different $A R$ of cylinder

\begin{tabular}{|c|c|}
\hline \multicolumn{2}{|c|}{ of cylinder } \\
\hline Number of blades & Optimal aspect ratio \\
\hline 1 & 3 \\
\hline 2 & 6 \\
\hline 3 & 9 \\
\hline 4 & 12 \\
\hline 5 & $12-15$ \\
\hline 6 & 15 \\
\hline 7 & 18 \\
\hline 8 & 21 \\
\hline 9 & 24 \\
\hline 10 & 27 \\
\hline
\end{tabular}

The proportionality coefficient which takes a value 3 is related to the blockage parameter $\beta=1 /(3 \pi)$ where:

$$
\beta=\mathrm{A}_{\text {blades }} / \mathrm{A}_{\text {disk }}=\mathrm{Nbd} / \pi \mathrm{R}_{\mathrm{t}}(1-\mathrm{Rht})
$$

Concluding here that with the chosen ratios of tip speed rotor and spinning cylinder, $\lambda=5$ and $\omega=0.5$, respectively, the Magnus wind turbine reaches the optimal efficiency when the ratio between the solid area occupied by the blades and the total disk area equals 3. This result is proven by Bychkov et al. (Bychkov et al. 2007, 2008a, b), in his configuration, where it is demonstrated experimentally that the most suitable for the Magnus wind turbine design is the use of 6 smooth rotating cylinders with an aspect ratio of 15 .

In the second step, the spinning cylinder ratio influence is examined with one of the optimal values of the aspect ratio, let be $A R=15$. The rotor tip speed ratio is fixed by the value $\lambda=5$. The results are reported in the Fig. 8 for the same previous values of number of blades. In the case where the number of blades is low $N b \leq 4$ and the level of spinning is relatively high; the calculations diverge and the power coefficient passes beyond the Betz limit which is physically unacceptable.

Besides, for the other values of the blades number the Magnus rotor efficiency falls with respect to the ideal limit of Betz except the two cases $N b=5$ and 6 which are represented in the enlarged view in Fig. 9.

In Fig. 9, the rotor with six cylinders appears relatively more performant than the other with five blades in the range of the spinning ratio limited almost between 0.4-0.7, while in the rest of the range, the turbine of five cylinders becomes the best. It is clear from the optimal results, for $N b=5$ and 6 , that the turbine power coefficient has low sensitivity to the change of the spinning ratio over the studied range 0.1-1.1.

Similarly, to analyze the effect of the rotor tip speed ratio for ten values of the blades number, the power coefficient is represented by fixing the relative speed of the column rotation and the aspect ratio as $\varpi=0.5$ and $A R=15$, respectively. The Fig. 10 shows

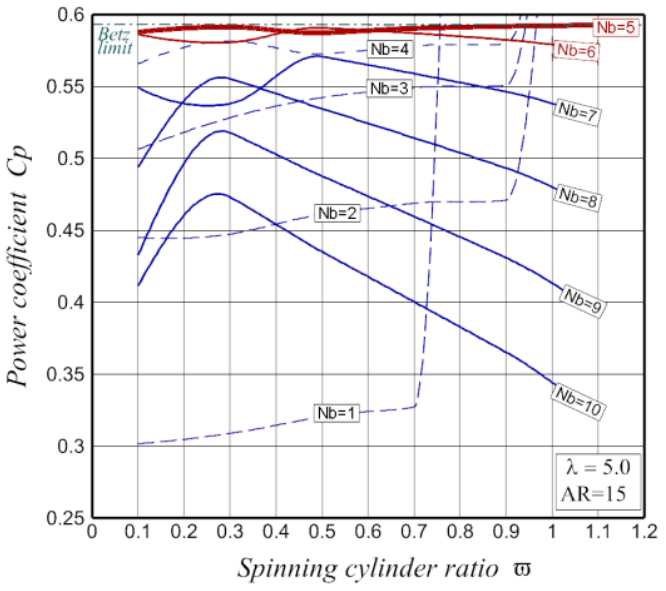

Fig. 8. $C p$ versus $\varpi$ for ten values of number of blades with $\lambda=5$ and $A R=15$.

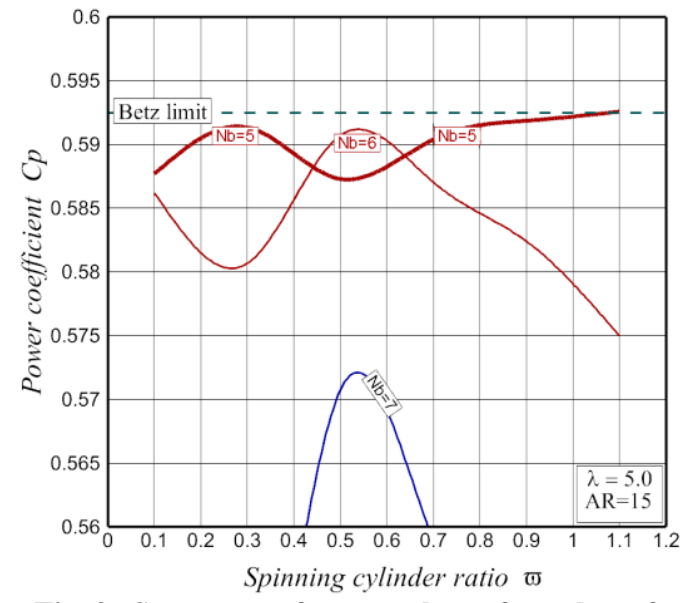

Fig. 9. $C p$ versus $\varpi$ for ten values of number of blades with $\lambda=5$ and $A R=15$ (enlarged view).

the operability range for this parameter. It seems that, for the above conditions with the number of blades greater than 7 and less than 3, the BEM code ceases to give results. Besides, between 3 and 7, the bell shape of the power coefficient curve appears and its top reaches the ideal limit.

Concerning the hub-tip ratio effect, the power coefficient is represented by fixing the relative speed of the column rotation, the tip speed ratio, the blade aspect ratio and the number of blades as $\varpi=0.5, \lambda=0.5, A R=15$ and $N b=5$, respectively. As it is shown in Fig. 11, this parameter marks its positive effect between the values 0.1 and 0.4 , which the power coefficient keeps almost the same value, and beyond the value 0.4 the wind turbine loses its high potential.

\section{CONCLUSION}

The Magnus type Horizontal Axis Wind Turbine MHAWT represents an innovative device and a promising type of the wind turbine used to overcome the problem of the low wind speed fields. This type of wind turbines uses a spinning cylinders 


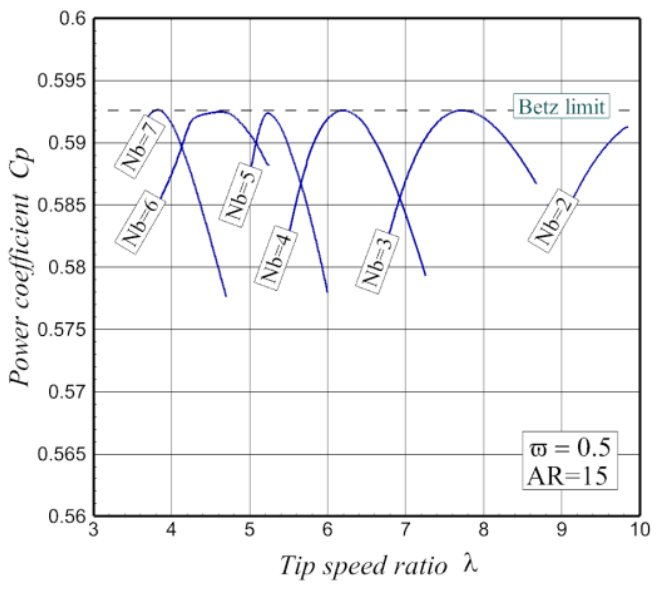

Fig. 10. $C p$ versus $\lambda$ for ten values of number of blades with $\lambda=0.5$ and $A R=15$.

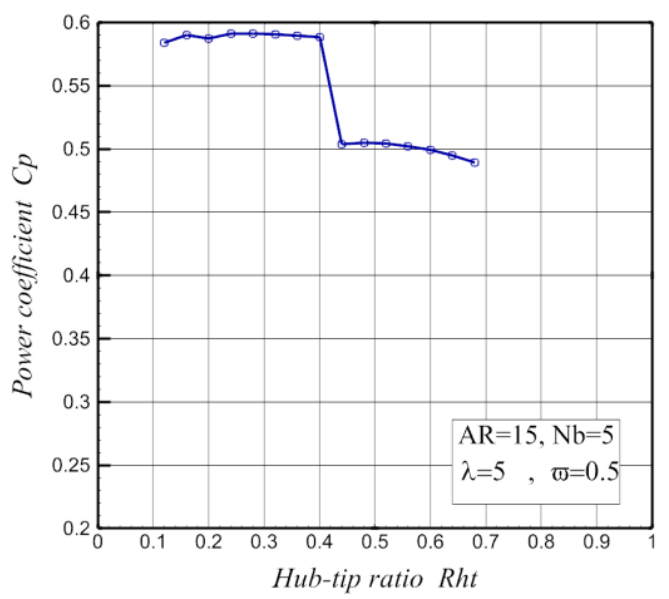

Fig. 11. $C p$ versus $R h t$ for the blade with $\lambda=5$, $A R=15, N b=15$ and $\varpi=0.5$.

instead the conventional blades and the lift force is generated under the Magnus effect. Among the more performant configurations of the MHAWT is especially when the spinning cylinders are equipped with spiral fins. In this current investigation, a parametric analysis is carried out on the Magnus wind turbine with spiral fins to assess its efficiency and identify the best geometric and kinematic parameters using the famous approach of the Blade Element Momentum BEM. The effects of the cylinder aspect ratio, the rotor tip speed ratio, the spinning column ratio and the hub tip ratio of the blade are successively studied for the number of blades varying from 1 to 10 . Concerning the BEM method, it was updated according the characteristics of this novel device. Therefore, the axial induction factor is assessed by solving a quadratic equation and the angular induction factor is calculated from a classic formulation including herein the term of spinning. The power coefficient that is used in this study to assess the performance of the MHAWT with spiral fins depicts the ideal aerodynamic behavior as the drag of the spinning cylinders is neglected. Concerning the geometric parameters, firstly for the blade aspect ratio effect, the wind turbine reaches the best efficiency for a solid area occupied by the blades equal a third of total rotor area. Secondly, the hub-tip ratio marks its positive effect between the values 0.1 and 0.4 , which the power coefficient maintains almost the same value, and outside this range the wind turbine loses its high potential. As for the kinematic parameters, the rotor has a low sensitivity to the spinning ratio corresponding to the optimal performance. The analyze of the rotor tip speed ratio leads to identify the operability range for this parameter, between 4 and 9 the bell shape of the power coefficient curve appears and its top reaches the ideal limit. Nevertheless, as this study is a preliminary analysis some other important factors related to the considered device push us to add further improvements such as the achievement of the experimental tests and 3D CFD simulation in order to validate the BEM results, identification of the impact of the drag coefficient, the insertion of the endplates at the tip of the cylinders, and the application of the multi-objective optimization, and all this, it aims to obtain high accurate results and well prediction of the real operating conditions.

\section{ACKNOWLEDGEMENTS}

The author would like to acknowledge the Algerian organism of research DGRSDT.

\section{REFERENCES}

Aneesh, D., S. Ronith, F. Romli, S. Bibin Sagaram and S. Suneesh (2016). Numerical analysis of Magnus wind turbine. $7^{\text {th }}$ International Conference on Mechanical and Aerospace Engineering, ICMA 2016, London UK.

Branlard, E. (2017). Wind Turbine Aerodynamics and Vorticity-Based Methods, Fundamentals and Recent Applications. Springer International Publishing, Switzerland.

Bychkov, N. (2008a). Magnus wind turbine. Thermophysics and Aeromechanics 15(2), 321331.

Bychkov, N., A. Dovgal and A. Sorokin (2008b). Parametric optimization of the Magnus wind turbine. International Conference on Methods of Aerophisical Research, ICMAR'08, Akademgorodok, Novosibirsk Russia.

Bychkov, N., A. Dovgal and V. Kovlov (2007). Magnus wind turbines as an alternative to the blade ones. The Science of Making Torque from Wind, IOP Publishing. Journal of Physics: Conference Series 75(2007)012004

Himri, Y., S. Himri and A. Stambouli (2010). Wind Speed Data Analysis used in Installation of Wind Energy Conversion Systems in Algeria. Transmission and Distribution Conference and Exposition (T\&D), 2010 IEEE PES, New Orleans, LA, USA.

Kheirandish, H. R. (2016). Magnus type wind power generator. Patent. US 9273666 B2.

Luo, D., D. Huang and G. Wu (2011). Analytical 
R. Mdouki et al. / JAFM, Vol. 14, No. 3, pp. 887-895, 2021.

solution on Magnus wind turbine power performance based on the blade element momentum theory. Journal of renewable and sustainable energy 3, 1-13.

Marzuki, O., A. Mohd Rafie, F. Romli, K. Ahmad and M. Abdul Hamid (2015). Torque performance study of Magnus wind turbine. International Review of Mechanical Engineering I.RE.M.E, 9 (1), 38-41.

Masaya, U., H. Hiroaki and I. Yuya (2018). Lift Enhancement of a rotating cylinder for Magnus wind turbine. Grand Renewable Energy Proceeding, Yokohama.

Murakami, N. (2007). Magnus type wind power generator. Patent. US 0046029 A1.

Murakami, N., H. Hiroaki, H. Toshihiro and N. Nasahide (2016). The mechanism of flow field around a rotating cylinder with fins for high performance Magnus wind turbine. Journal of Energy and Power Engineering 10(1), 23-31.
Sedaghat, A. (2014). Magnus type wind turbines: Prospectus and challenges in design and modelling. Journal of Renewable Energy 62, 619-628.

Seifert, J. (2012). A review of the Magnus effect in aeronautics. Progress in Aerospace Sciences. $55,17-45$.

Sørensen, J., N. (2016). General Momentum Theory for Horizontal Axis Wind Turbines. Springer International Publishing, Switzerland.

Tahani, M. and M. Moradi (2016). Aerodynamic investigation of a Wind Turbine using CFD and Modified BEM Methods. Journal of Applied Fluid Mechanics 10(1), 23-31.

Xiaojing, S., Z. Yueqing and C. Yang and H. Dianghi (2012). A three-dimensional numerical study of the Magnus wind turbine with different blade shapes. Journal of Renewable and Sustainable Energy 4, 1-14. 